8. Tambyah PA, Maki DG. The relationship between pyuria and infection in patients with indwelling urinary catheters: a prospective study of 761 patients. Arch Intern Med 2000;160: 673-677.

9. Humphries RM, Dien Bard J. Point-counterpoint: reflex cultures reduce laboratory workload and improve antimicrobial stewardship in patients suspected of having urinary tract infections. J Clin Microbiol 2016;54:254-258.

10. Fakih MG, Krein SL, Edson B, Watson SR, Battles JB, Saint S. Engaging health care workers to prevent catheter-associated urinary tract infection and avert patient harm. Am J Infect Control 2014;42:S223-S229.

\section{Detection of Piperacillin-Tazobactam- Resistant/Pan- $\beta$-Lactam-Susceptible Escherichia coli with Current Automated Susceptibility Test Systems}

To the Editor-The determination of phenotypic antimicrobial resistance via currently available automated susceptibility systems is well established worldwide. Phenotypic testing is continuously challenged by ever-changing alterations in gene expression, genetic mutation, or new gene acquisition from another bacterium. ${ }^{1}$ The development of antibiotic resistance increases the risk of clinical failure in infected patients, especially if such resistance is unknown to the clinical practitioner. $^{2}$ The global use of automated microbiology test systems, such as MicroScan (Beckman Coulter, Brea, CA), Phoenix (Becton Dickinson Diagnostic Systems, Sparks, MD), and Vitek 2 (bioMérieux, Durham, NC), for the identification and antimicrobial susceptibility testing (AST) of bacteria has grown, but these systems have had serious reporting errors with certain organism-antibiotic combinations. ${ }^{3-5}$ We have recently identified 43 Escherichia coli isolates from 29 US hospitals that are pan- $\beta$-lactam-susceptible (ie, all cephalosporins, monobactams and carbapenems [PBL-S]) but are resistant to piperacillin-tazobactam (TZP-R), a broadspectrum $\beta$-lactamase inhibitor. ${ }^{6-8}$ In this study, we assessed the accuracy of the aforementioned systems in determining the susceptibility profile of this unique phenotype.

We sent 14 unidentified clinical isolates of E. coli, 4 piperacillin-tazobactam susceptible (TZP-S)/PBL-S and 10 genotypically confirmed TZP-R/PBL-S to 3 sites for AST using MicroScan, Phoenix, and Vitek 2. To assess the accuracy of the categorical results provided by these systems (ie, susceptible, intermediate, or resistant), piperacillin-tazobactam minimum inhibitory concentrations (MICs) were determined in triplicate by broth microdilution (BMD) according to the 2016 Clinical Laboratory Standards Institute guidelines. AST data were
TABLE 1. In vitro Susceptibility profile of E. coli Against Piperacillin-Tazobactam Using Broth Microdilution (BMD) and 3 Automated Susceptibility Test Systems

\begin{tabular}{|c|c|c|c|c|}
\hline \multirow[b]{2}{*}{ E. coli } & \multicolumn{4}{|c|}{ Phenotypic Profile Method ${ }^{\mathrm{a}}$} \\
\hline & BMD (TZP MIC) & MicroScan & Phoenix & Vitek 2 \\
\hline EC C1-6 & S (16) & $S$ & $S$ & S \\
\hline EC C2-9 & $\mathrm{R}(512)$ & I & $\mathrm{R}$ & $\mathrm{R}$ \\
\hline EC C3-23 & $\mathrm{R}(\geq 2048)$ & $\mathrm{R}$ & $\mathrm{R}$ & $\mathrm{R}$ \\
\hline EC C1-7 & S (4) & $\mathrm{S}$ & $\mathrm{S}$ & $S$ \\
\hline EC C1-23 & $S(16)$ & $S$ & $\mathrm{~S}$ & $\mathrm{~S}$ \\
\hline EC C6-25 & R (2048) & $\mathrm{R}$ & $\mathrm{R}$ & $\mathrm{R}$ \\
\hline EC C7-1 & $\mathrm{R}(256)$ & $\mathbf{S}$ & $\mathbf{I}$ & I \\
\hline EC C10-11 & $\mathrm{R}(\geq 2048)$ & I & $\mathrm{R}$ & $\mathrm{R}$ \\
\hline EC C11-14 & $\mathrm{R}(\geq 2048)$ & $\mathrm{R}$ & $\mathrm{R}$ & $\mathrm{R}$ \\
\hline EC C2-5 & S (4) & $\mathrm{S}$ & $\mathrm{S}$ & $\mathrm{S}$ \\
\hline EC C12-1 & $\mathrm{R}(512)$ & $\mathbf{S}$ & $\mathrm{R}$ & $\mathbf{I}$ \\
\hline EC C14-26 & $\mathrm{R}(\geq 2048)$ & $\mathrm{R}$ & $\mathrm{R}$ & $\mathrm{R}$ \\
\hline EC C18-6 & $\mathrm{R}(\geq 2048)$ & $\mathrm{R}$ & $\mathrm{R}$ & $\mathrm{R}$ \\
\hline EC C30-5 & $\mathrm{R}(256)$ & I & $\mathrm{R}$ & $\mathrm{R}$ \\
\hline
\end{tabular}

NOTE. TZP, piperacillin-tazobactam; EC, Escherichia coli; MIC, minimum inhibitory concentration $(\mu \mathrm{g} / \mathrm{mL}), \mathrm{S}$, susceptible; I, intermediate; $\mathrm{R}$, resistant.

${ }^{\mathrm{a}}$ Data shown in bold are erroneous results.

determined via specific manufacturer and laboratory guidelines for each system. Categorical errors reported by the automated systems in relation to BMD were classified as very major (false susceptibility), major (false resistance), or minor (involving the intermediate category interpretation). ${ }^{9}$

The MICs of these isolates against piperacillin-tazobactam and the interpretive classification generated by each system are reported in Table 1. Notably, none of the systems demonstrated $100 \%$ accuracy in reporting the phenotypic profile when compared with the BMD reference method. Phoenix reported 1 minor error, Vitek 2 demonstrated 2 minor errors, and MicroSca produced the most inconsistent results, with 2 very major errors and 3 minor errors.

These findings are relevant considering that piperacillintazobactam is used empirically in compromised hosts or as directed therapy for E. coli infections given the retention of high susceptibility rates compared with other available antibiotics. ${ }^{6,10}$ Therefore, the detection of this TZP-R/PBL-S phenotype is vital to providing appropriate antimicrobial therapy and optimal patient care. Furthermore, the use of cascade reporting has been implemented in many hospitals to control antibiotic use, which often involves reporting the susceptibilities of broad-spectrum agents only when the organism is resistant to more narrow-spectrum agents. Therefore, cascade reporting may misrepresent the susceptibility of this organism if it is overlooked due to its susceptibility to more narrow-spectrum antimicrobial agents. Although further studies are needed to determine the clinical relevance of these TZP-R/PBL-S strains, the high use of piperacillin-tazobactam and the prevalence of $E$. coli infections make the recognition of this phenotype 
imperative because current AST systems may not accurately characterize the resistance profile. Moreover, the interpretation of AST outputs should be undertaken with caution, especially in the setting of cascade reporting.

\section{ACKNOWLEDGMENTS}

Financial support: This study was internally funded by the Center for Anti-Infective Research and Development, Hartford Hospital, Hartford, Connecticut.

Potential conflicts of interest: All authors report no conflicts of interest relevant to this article.
7. Mediavilla JR, Schneider Z, Nwaigwe C, et al. Molecular characterization of cephalosporin/carbapenem/monobactam susceptible but piperacillin-tazobactam (TZP) resistant E. coli. Presented as Abstract 1181 at: ID Week, October 7-11, 2015, San Diego, CA.

8. Monogue ML, Nicolau DP. In vitro-in vivo discordance with humanized piperacillin-tazobactam exposures against piperacillintazobactam resistant/pan- $\beta$-lactam-susceptible Escherichia coli. Antimicrob Agents Chemother 2016. doi: 10.1128/AAC.01208-16.

9. Rhodes NJ, Richardson CL, Heraty R, et al. Unacceptably high error rates in Vitek 2 testing of cefepime susceptibility in extended-spectrum- $\beta$-lactamase-producing Escherichia coli. Antimicrob Agent Chemother 2014;58:3757-3761.

10. Ng TM, Khong WX, Harris PN, et al. Empiric piperacillintazobactam versus carbapenems in the treatment of bacteraemia due to extended-spectrum beta-lactamase-producing Enterobacteriaceae. PLoS ONE 2016;11:e0153696.

\begin{abstract}
Affiliations: 1. Center for Anti-infective Research and Development, Hartford Hospital, Hartford, Connecticut; 2. Department of Microbiology, Eastern Connecticut Health Network, Manchester, Connecticut; 3. Department of Microbiology, Veterans' Affairs Boston Health Care System, West Roxbury, Massachusetts; 4. Pathology and Laboratory Medicine, Boston University School of Medicine, Boston, Massachusetts; 5. Department of Microbiology, Hartford Hospital, Hartford, Connecticut; 6. Division of Infectious Diseases, Hartford Hospital, Hartford, Connecticut

Address correspondence to David P. Nicolau, PharmD, FCCP, FIDSA, Center for Anti-infective Research and Development, Hartford Hospital, 80 Seymour Street, Hartford, CT 06102 (david.nicolau@hhchealth.org). Infect Control Hosp Epidemiol 2017;38:379-380

( 2017 by The Society for Healthcare Epidemiology of America. All rights reserved. 0899-823X/2017/3803-0024. DOI: 10.1017/ice.2016.325
\end{abstract}

\section{REFERENCES}

1. Martinez JL, Baquero F. Mutation frequencies and antibiotic resistance. Antimicrob Agent Chemother 2000;44:1771.

2. Wang Q, Zhang Y, Yao X, et al. Risk factors and clinical outcomes for carbapenem-resistant Enterobacteriaceae nosocomial infections. Eur J Clin Microbiol Infect Dis 2016;35:1679-1689.

3. Sader HS, Fritsche TR, Jones RN. Accuracy of three automated systems (MicroScan WalkAway, VITEK, and VITEK 2) for susceptibility testing of Pseudomonas aeruginosa against five broad-spectrum beta-lactam agents. J Clin Microbiol 2006;44: 1101-1104.

4. Gordon NC, Wareham DW. Failure of the MicroScan WalkAway system to detect heteroresistance to carbapenems in a patient with Enterobacter aerogenes bacteremia. J Clin Microbiol 2009; 47:3024-3025.

5. Kulah C, Aktas E, Comert F, Ozlu N, Akyar I, Ankarali H. Detecting imipenem resistance in Acinetobacter baumannii by automated systems (BD Phoenix, Microscan WalkAway, Vitek 2); high error rates with Microscan WalkAway. BMC Infect Dis 2009;9:30.

6. Sutherland CA, Nicolau DP. Susceptibility profile of ceftolozane/ tazobactam and other parenteral antimicrobials against Escherichia coli, Klebsiella pneumoniae, and Pseudomonas aeruginosa from US Hospitals. Clin Ther 2015;37:1564-1571.

\section{Pathogen and Procedure Trends Among Surgical-Site Infections at a Children's Hospital: A 20-Year Experience}

To the Editor-Surgical-site infections (SSIs) are common healthcare-associated infections that increase patient morbidity and mortality and cost the US healthcare system billions of dollars annually. ${ }^{1}$ The 1999 Centers for Disease Control and Prevention (CDC) SSI prevention guidelines define a set of recommendations based on relative pathogen frequency and patient- and procedure-based SSI risk known at that time. ${ }^{2}$ Most of the effort in SSI prevention has been built around these guidelines since their publication. ${ }^{3,4}$ Additional recommendations have been published to direct specific aspects of SSI prevention, such as antimicrobial prophylaxis, in addition to their implementation and tracking. ${ }^{5,6}$ While these updated guidelines have included new data, they are built upon the foundation of the 1999 CDC guidelines. Despite SSI rate improvement, SSIs remain the most common and costly healthcare-acquired infection in the United States. ${ }^{1}$

We hypothesized that targeted SSI prevention efforts based on the 1999 guidelines could have changed the relative pathogen frequency, possibly indicating a need to refine our approach to SSI prevention. We used SSI data from our medical center over 2 decades to study trends in SSI pathogen frequency.

\section{METHODS}

Pathogens associated with SSIs in incision class I and II surgical procedures ${ }^{2}$ performed between January 1, 1994, and December 31, 2015, were obtained through the Infection Prevention and Control Program at Cincinnati Children's 